\title{
Transcription strategies related to photosynthesis and nitrogen metabolism of wheat in response to nitrogen deficiency
}

\author{
Xin $\mathrm{Liu}^{1,2^{*}+}$, Chengmiao $\mathrm{Yin}^{3+}$, Li Xiang ${ }^{3}$, Weitao Jiang ${ }^{3}$, Shaozhuo $\mathrm{Xu}^{3}$ and Zhiquan Mao ${ }^{3+}$
}

\begin{abstract}
Background: Agricultural yield is closely associated with nitrogen application. Thus, reducing the application of nitrogen without affecting agricultural production remains a challenging task. To understand the metabolic, physiological, and morphological response of wheat (Triticum aestivum) to nitrogen deficiency, it is crucial to identify the genes involved in the activated signaling pathways.

Results: We conducted a hydroponic experiment using a complete nutrient solution (N1) and a nutrient solution without nitrogen (NO). Wheat plants under nitrogen-deficient conditions (NDC) showed decreased crop height, leaf area, root volume, photosynthetic rate, crop weight, and increased root length, root surface area, root/shoot ratio. It indicates that nitrogen deficiency altered the phenotype of wheat plants. Furthermore, we performed a comprehensive analysis of the phenotype, transcriptome, GO pathways, and KEGG pathways of DEGs identified in wheat grown under NDC. It showed up-regulation of Exp (24), and Nrt (9) gene family members, which increased the nitrogen absorption and down-regulation of Pet (3), Psb (8), Nar (3), and Nir (1) gene family members hampered photosynthesis and nitrogen metabolism.
\end{abstract}

Conclusions: We identified 48 candidate genes that were involved in improved photosynthesis and nitrogen metabolism in wheat plants grown under NDC. These genes may serve as molecular markers for genetic breeding of crops.

Keywords: Nitrogen deficiency, Nitrogen metabolism, Photosynthesis, Transcriptome, Wheat

\section{Background}

Excessive nitrogen application and low nitrogen utilization efficiency in winter wheat crops are challenging tasks across the world [1]. The low nitrogen utilization efficiency in wheat is primarily due to the excessive application of nitrogen fertilizer [2]. Besides, it causes environmental pollution and hampers the sustainable development of agriculture. On the premise of

\footnotetext{
* Correspondence: liux@sdau.edu.cn

Xin Liu and Chengmiao Yin are both the first authors.

'Zhiquan Mao has the equal contribution as Xin Liu.

'State Key Laboratory of Crop Biology, College of Agronomy, Shandong

Agricultural University, Taian 271018, Shandong, China

${ }^{2}$ ShanDong Shofine Seed Technology Co., Ltd., Jiangxiang 272400,

Shandong, China

Full list of author information is available at the end of the article
}

ensuring crop yield, reduced nitrogen application demands an urgent investigation. An in-depth understanding of physiological, metabolic, and morphological processes in wheat using molecular breeding methods can improve crop yield and nitrogen use efficiency (NUE) $[3,4]$ in wheat plants grown under nitrogendeficient conditions (NDC).

A detailed understanding of the plant's physiology, metabolism, and root canopy structure is crucial for improving crop yield and resource utilization efficiencies under stress conditions, such as shading, drought, or nutrition deficiency [5-7]. As shown in a previous study, reduced nitrogen application modified the root morphology and improved root architecture, which in turn increased the nitrogen absorption capacity and NUE [8], 
but it reduced the photosynthesis and metabolic rate [9, 10]. Thus, to improve the adaptability of the wheat plant to nitrogen deficiency, it is crucial to discern the physiological and metabolic processes of the wheat plant at the transcriptomic level.

Nitrogen deficiency alters the gene expression in plants. Nitrogen deficiency in barley plants induced the upregulation of HvNiR1, HvGS2, HvGLU2, downregulation of HvASN1 in the shoot, and upregulation of HvGLU2 in the root. Thus, it improved the adaptability of barley plants to nitrogen-deficiency [11]. The upregulated alternative oxidase (AOX) increased the utilization of excessive sugar and balanced the carbon level under NDC [12-14]. Similarly, the GmCZ-SOD1 gene was highly induced in the roots of the soybean plant grown under NDC [2]. 1799 differentially expressed genes (DEGs) were identified in maize crops grown under NDC [11]. Although multiple transcriptomic studies have been performed on the wheat crop, genes associated with wheat crop's physiology and metabolism under NDC remain unknown, demanding an in-depth investigation [15].
This study therefore conducted experiment which aimed to: (i) explore the physiological, metabolic and morphological changes of wheat under nitrogen deficiency condition; (ii) screen the differentially expressed genes (DEGs) from wheat transcriptome under nitrogen deficiency; (iii) after comprehensive analysis of transcription, metabolic pathway and phenotype of important physiological and metabolic processes, we try to find out the potential genes which can be promote wheat growth under nitrogen deficiency.

\section{Result}

Morphological and physiological changes in wheat grown under the nitrogen-deficient condition

The altered morphological and physiological states of wheat are depicted in Fig. 1. The height of the wheat plant in the N0 group was 0.75 times significantly lower than the wheat plants in the N1 group (Fig. 1a). The leaf area per plant of the wheat plants in the NO group was 0.70 times significantly smaller than the wheat plants in the N1 group. However, no significant differences were observed in the specific leaf area of wheat plants in the N0 and N1 groups. The net photosynthetic rate (Pn)
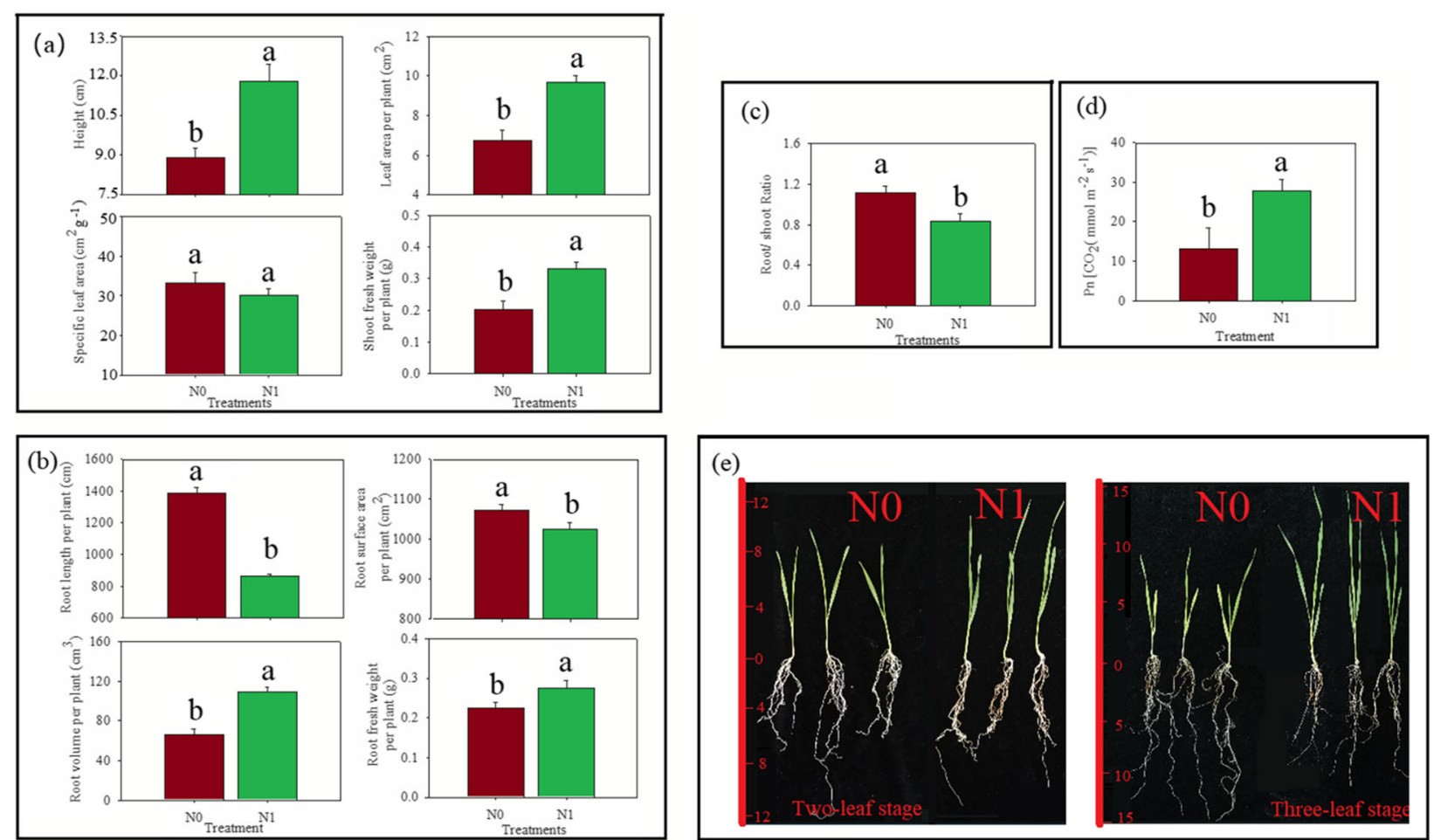

Fig. 1 Effects of nitrogen content on winter wheat crop. a The shoot morphology, including crop height, leaf area per plant, specific leaf area, shoot fresh weight; $\mathbf{b}$ root morphology, including root length per plant, root surface area, root volume per plant, root fresh weight per plant; $\mathbf{c}$ root/shoot ratio; d net photosynthetic rate; e phenotypes, under normal nitrogen (N1) and nitrogen-deficient (N0) conditions. The root length per plant, root surface area, root volume per plant, root fresh weight per plant were the sum of all roots of one plant. Significance levels of differences between N0 and N1 group were estimated using the two-tailed t-test method. Different lowercase letters represent significant differences 
and fresh shoot weight of wheat plants in the N0 group were 0.47 and 0.61 times significantly lower, respectively, than the wheat plants in the N1 group (Fig. 1d). It showed that nitrogen deficiency led to reduced crop height, leaf area per plant, Pn, and fresh shoot weight in wheat plants.

The root length per plant of wheat plants in the NO group was 1.61 times significantly more than wheat plants in the N1 group (Fig. 1b). Besides, the root volume per plant of wheat plants in the N0 group was 0.61 times lower than wheat plants in the N1 group. However, the root surface area per plant, fresh root weight, root shoot ratio of wheat plants in the N0 group was $1.04,0.82$, and 1.36 times higher, respectively, than wheat plants in the N1 group (Fig. 1c). It showed that nitrogen deficiency resulted in an increased root length, root surface area per plant, fresh root weight, root shoot ratio, and reduced root volume per plant.

\section{Global analysis of RNA-seq data of wheat plants grown under the nitrogen-deficient condition}

The number of genes expressed in different parts of N0 group wheat plants was calculated to construct the stacked histogram (Fig. S1a). A total of 72,487-78,729 genes were identified in the wheat shoot of the N0 group, out of which 17,116-22,418 genes had FPKM (Fragments Per Kilobase of transcript per Million fragments mapped) values $>1$. Besides, 63,273-64,413 genes were identified in the wheat root of the N0 group, out of which 27,785-29,233 genes had FPKM values $>1$.

Principal component analysis (PCA) was applied to explore the relationship between samples by locating the samples at different dimensions (Fig. S1b). Less clustering distance indicated more identical samples. PCA1 reflected the difference in root and shoot, accounting for $99.41 \%$ of the total variation. Besides, shoot and root transcription differences between the $\mathrm{N} 0$ and $\mathrm{N} 1$ groups of wheat plants were deduced by PCA2 and PCA3, which accounted for 0.21 and $0.11 \%$ of the total variation. PCA3 reflected the root transcription difference between the N0 and N1 groups of wheat plants, accounting for a total variation of $0.11 \%$.

The volcanogram (Fig. 2a, b) and cluster map (Fig. 2c, d) of $p$-values and $\log _{2} \mathrm{FC}$ were applied to screen the differentially expressed genes (DEGs) in the No group of wheat plants as compared to the control (N1) wheat plants. We identified a total of 3949 DEGs in the shoots of wheat plants grown under NDC, out of which 1535 were up-regulated, and 2414 were down-regulated. Besides, we identified a total of 3911 DEGs in roots of wheat plants grown under NDC, 1236 of which were upregulated, and 2675 were down-regulated (Fig. 2e). The Venn map (Fig. 2f) revealed that 1535 DEGs were upregulated and 2414 were down-regulated in both shoot and root of wheat plants grown under NDC, and a total of 372 DEGs were identified in roots and shoot.

\section{Functional analysis of DEGs identified in wheat grown under the nitrogen-deficient condition}

1205 up-regulated genes and 1888 down-regulated genes in shoots, while 961 up-regulated genes and 1883 downregulated genes identified in roots of wheat plants grown under NDC, were enriched in Gene Ontology (GO) analysis (Fig. S2). The enriched genes were classified into 3 major classes and 64 sub-classes, and some of these genes belonged to two or more categories. Cellular process, metabolic process, binding, and catalytic activity were the top enriched categories, which included more than 980 DEGs (Table 1).

We performed KEGG pathway enrichment analysis of (Fig. 3a, b) DEGs from shoots and roots of wheat plants grown under NDC, and the pathways that showed enrichment of the highest number of DEGs are discussed here. Root DEGs showed enrichment of the gene information processing-translation pathway (142 downregulated genes), and metabolism-biosynthesis of other secondary metabolites pathway (54 up-regulated genes). Root DEG's KEGG pathway analysis led to the enrichment of the metabolism-carbohydrate metabolism pathway (118 down-regulated genes) and the metabolismbiosynthesis of other secondary metabolites pathway (78 up-regulated genes). Shoot DEG's KEGG pathway analysis showed enrichment of monobactam biosynthesis (Fig. 3c) and the nitrogen metabolism pathway (Fig. 3d).

\section{Analysis of gene families associated with cellular process} Expansin family members primarily belong to the GO category-cellular process. 3 DEGs in shoot of wheat plant grown under NDC belonged to (Fig. 4) expansin family, including TreasCS2B02G411700 (up-regulated), TreasCS1A02G30020 (down-regulated) and TreasCS1B02G310300 (down-regulated). Also, 6 down-regulated genes (TreasCS6A02G307900 and so on) and 24 up-regulated genes (TreasCS5B02G528400 and so on) in roots of the wheat plant grown under NDC belonged to the expansin family.

\section{Analysis of gene families associated with metabolic process}

Pet and Psb family members serve as crucial photosystem members in the wheat shoot and belong to the GO category-metabolic process. In wheat plants grown under NDC, 3 down-regulated DEGs (Fig. 5) belonged to the Pet family (TreasCS7A02G325500 and so on), 8 down-regulated DEGs belonged to the Psb family (TreasCS3D02G523300 and so on), and 1 up-regulated DEG belonged to the Psb family (TreasCS6B02G412100). 

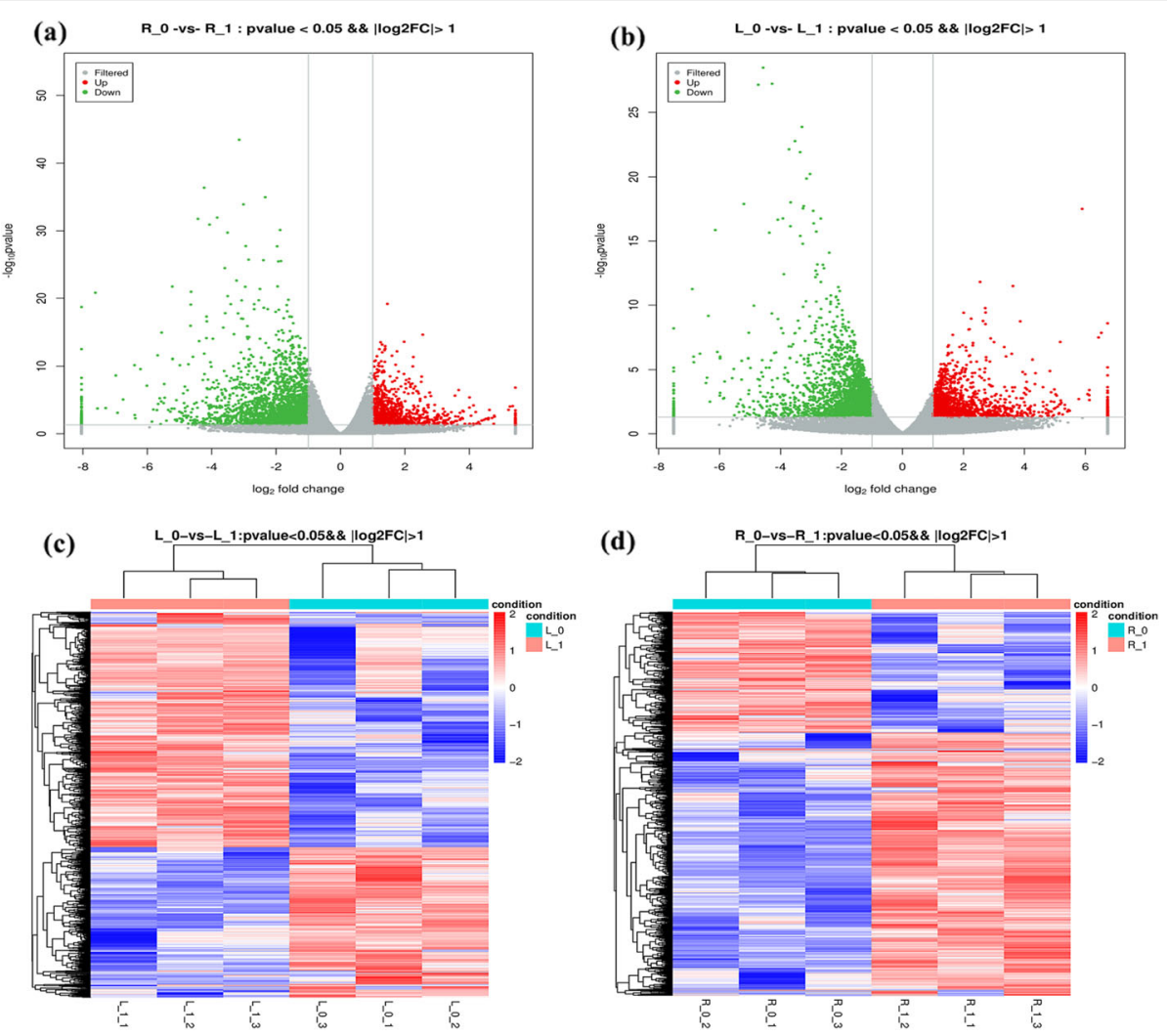

(e)

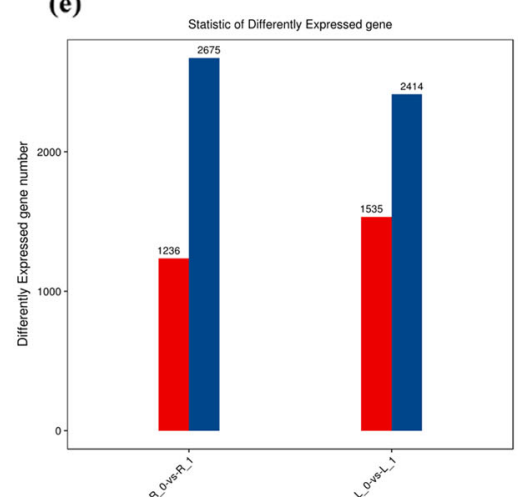

(f)

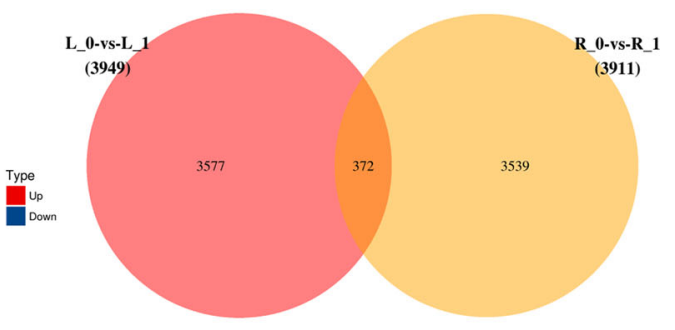

Fig. 2 Volcanogram (a represents root, b represents shoot), cluster map (c indicates shoot, $d$ indicates root), e number of differentially expressed genes in wheat, and $\mathbf{f}$ Venn map under nitrogen-deficient condition. R_0 and L_0 represent the root and shoot of N0 (nutrition solution without nitrogen) group of plants, respectively; R_1 and L_1 represent the root and shoot of N1 (complete nutrition solution) group of plants, respectively. In volcanogram $(\mathbf{a}, \mathbf{b})$, gray points were the genes with a non-significant difference, red and green points were the genes with significant differences; $X$-axis display of $\log _{2}$ foldchange $(F C)$, and $Y$-axis display $p$-value. In the cluster map (c, $\mathbf{d}$ ), red represent up-regulated and blue represent down-regulated protein-coding genes

Nar and Nrt family members are involved in nitrogen metabolism and belong to the GO category-metabolic process. In wheat plants grown under NDC, 3 downregulated genes from root and shoot (Fig. 6) belonged to Nar family (TreasCS6A02G326200, TreasCS6B02G356800, and TreasCS6D02G306000), 2 up-regulated genes from root belonged to Nar family members (TreasCS6A02G210000 and TreasCS6D02G193100), and 9 up-regulated DEGs from root belonged to Nrt family (TreasCS6A02G031100). 
Table 1 The number of differentially expressed genes (DEGs) in the four pathways with the largest number of genes under the nitrogen-deficient condition

\begin{tabular}{lllll}
\hline GO category & Sub-category & DEGs-Up & DEGs-Down \\
\hline Shoot & Biological process & Cellular process & 385 & 947 \\
& Biological process & Metabolic process & 498 & 1029 \\
& Molecular function & Binding & 672 & 1031 \\
\cline { 3 - 4 } Root & Molecular function & Catalytic activity & 560 & 854 \\
& Biological process & Cellular process & 312 & 669 \\
& Biological process & Metabolic process & 390 & 891 \\
& Molecular function & Binding & 527 & 893 \\
\cline { 3 - 4 } & Molecular function & Catalytic activity & 429 & 936 \\
\hline
\end{tabular}

\section{Validation of transcriptomic data}

As per the RT-qPCR based validation study, expression levels of 94 (46 shoot genes and 48 root genes) out of 100 candidate genes were in line with the FPKM values of transcriptomic data (Fig. 7a). It showed that around $94 \%$ of the transcriptomic data were reliable. The coefficients of $\mathrm{X}$ of regression lines were 0.93 and 1.05 for shoot and root, respectively, which indicated high accuracy of the transcriptomic data. The RT-qPCR data of 50 candidate genes from root and shoot are depicted in Fig. $7 \mathrm{~b}$, and Fig. 7c, respectively. The comparison between RT-qPCR and transcriptomic data of each gene can be queried using Table S1 and Table S2.

\section{Discussion}

The altered morphology, metabolism, and physiology of wheat plants can be inferred from its transcriptomic data [16-18]. As per a previous report, in the photosynthesis

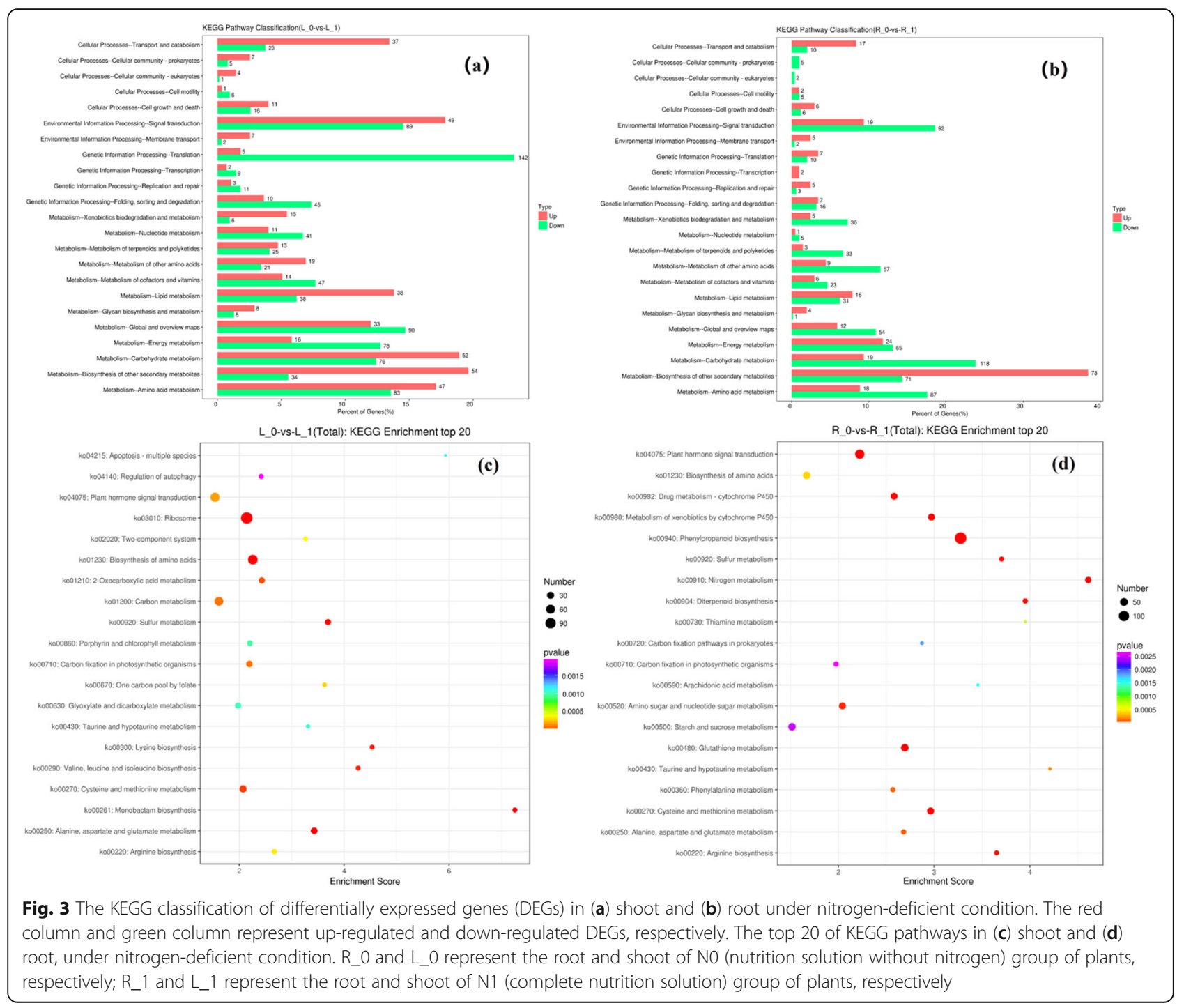




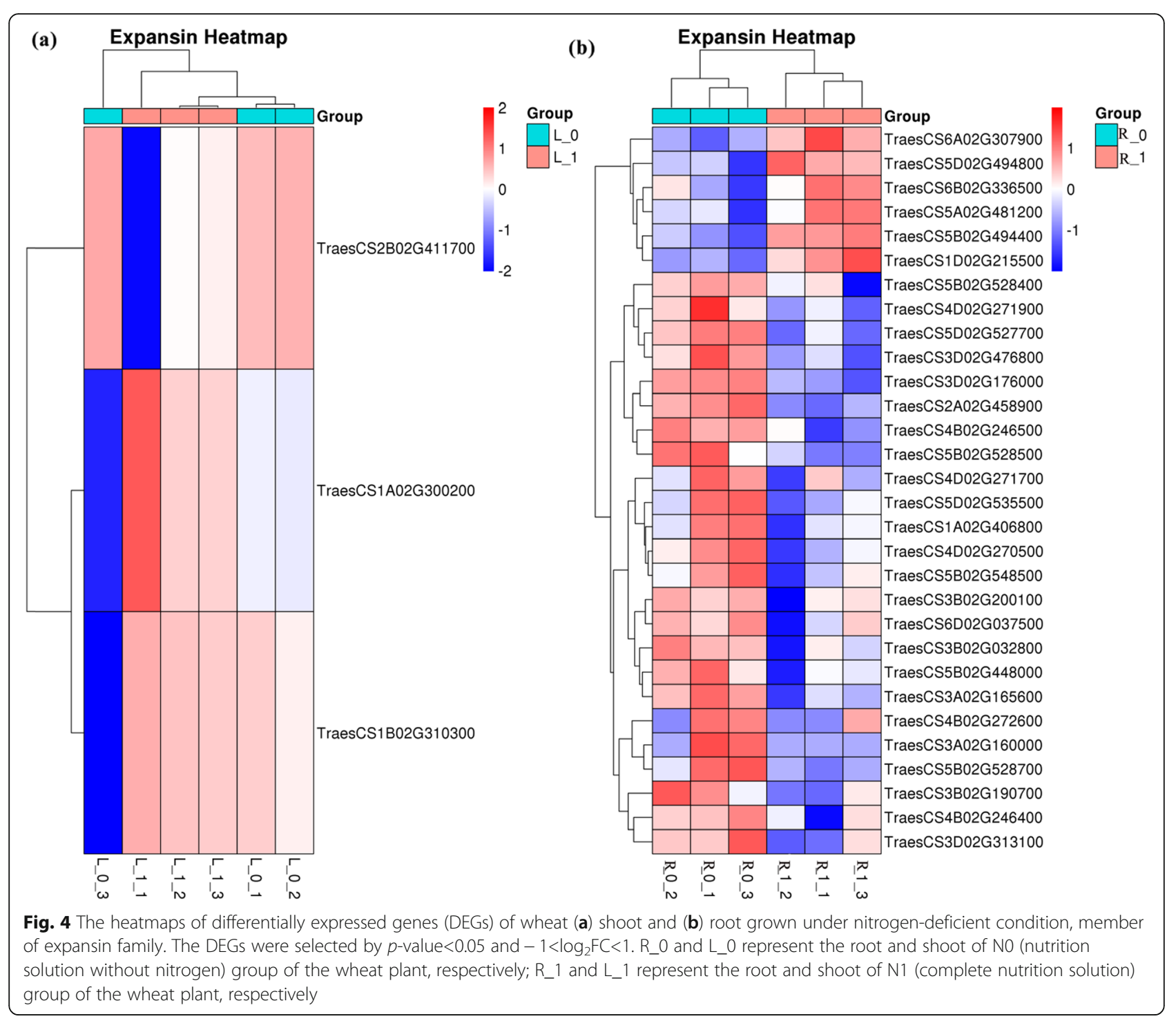

pathway (Fig. 8a), the proteins coded by the Pet and Psb gene families were crucial components of cytochrome b6/f complex, photosynthetic electron transport, and photosystem II [19-23]. Previous studies showed that the inhibition of these proteins hampered the photosynthetic efficiency of plants [24, 25]. In the current study, genes belonging to Pet and Psb gene families were found to be downregulated. These down-regulated genes led to the inhibition of photosynthetic electron transport and the photosystem II pathway in wheat plants grown under NDC, which reduced the photosynthetic rate and energy metabolism.

Furthermore, DEGs identified in wheat plants grown under NDC were also enriched in the nitrogen metabolism pathway (Fig. 8b, d). DEGs belonging to Nar (nitrate reductase) gene family were involved in the nitrate-N reduction to nitrite- $\mathrm{N}$ process $[26,27]$. DEGs belonging to Nir (Nitrite reductase) gene family were involved in the nitrite- $\mathrm{N}$ reduction to the ammonium- $\mathrm{N}$ process $[28,29]$. Moreover, DEGs belonging to Nrt gene family were involved in the process of nitrogen transport from extracellular to intracellular process [30]. Moreover, as per the previous report, the Nrt family were found to be involved in root growth, flowering time, and transcriptional regulation of multiple physiological processes, hormonal and nitrate signaling [31-34]. The up-regulated DEGs, the member of Nir and Nrt gene families, increased the nutrient uptake in crops [35-37]. The expression levels of genes identified in the shoot of wheat grown under NDC, which belonged to Nar and Nir gene families, and the component of the nitrogen metabolism pathway was found to be down-regulated. Similarly, in the root, the expression levels of genes belonging to the Nir gene family were found to be down-regulated, which in turn mitigated the nitrogen metabolism pathway in both shoot and root. Interestingly, the expression of Nrt gene family members 


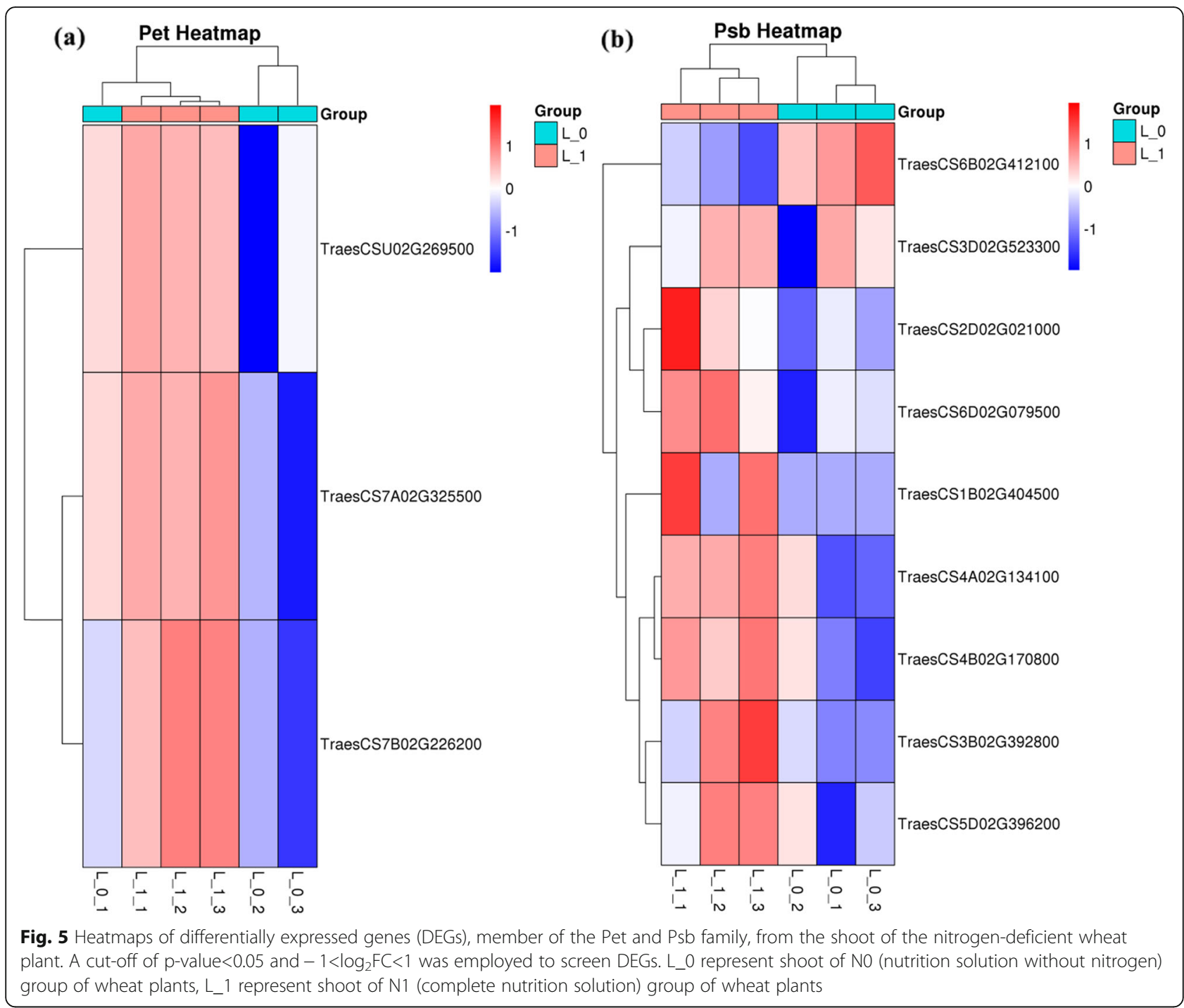

in nitrogen-deficient wheat root was up-regulated, which accelerated the movement of extracellular nitrogen into cells. The highest enrichment score of nitrogen metabolism pathway in wheat plant grown under NDC indicated that transcription differences had the highest influence on root nitrogen metabolism.

In the extracellular region pathway (Fig. 8c), the expansin gene family member increased the extensibility of the plant cell wall [38-40]. Previous studies stated that overexpressed expansin gene family members altered the crop morphology and improved the adaptability of crops to stress or low nutrition [41, 42]. For instance, the overexpressed TaEXPB23 altered the root system architecture of transgenic tobacco plants and improved the adaptability of plants to low phosphorus conditions [8]. In this study, under the NDC, the expression of expansin gene family members in root was upregulated, which led to increased root length and surface area of wheat plants. The increased root length and surface area increased the nitrogen absorption efficiency of the wheat plants grown under NDC.

The transcription level of plants changes in accordance with the external environmental conditions [39, 43], which in turn affect the protein levels and metabolism process, culminating in matter accumulation and morphological changes $[8,44]$. Some responses improve the adaptability of crops to the external environment. Moreover, the differential expression of genes in roots and leaves leads to different effects. The up-regulated expression of genes belonging to expansin and Nrt gene families that were identified in the root of wheat grown under NDC were involved in increasing the root surface area and nitrogen transport. It can be regarded as the adaptation of wheat plants to increase nitrogen absorption (Fig. 9).

The expression of Pet, Psb, Nar, Nir gene family members were found to be down-regulated, which inhibited the rate of 


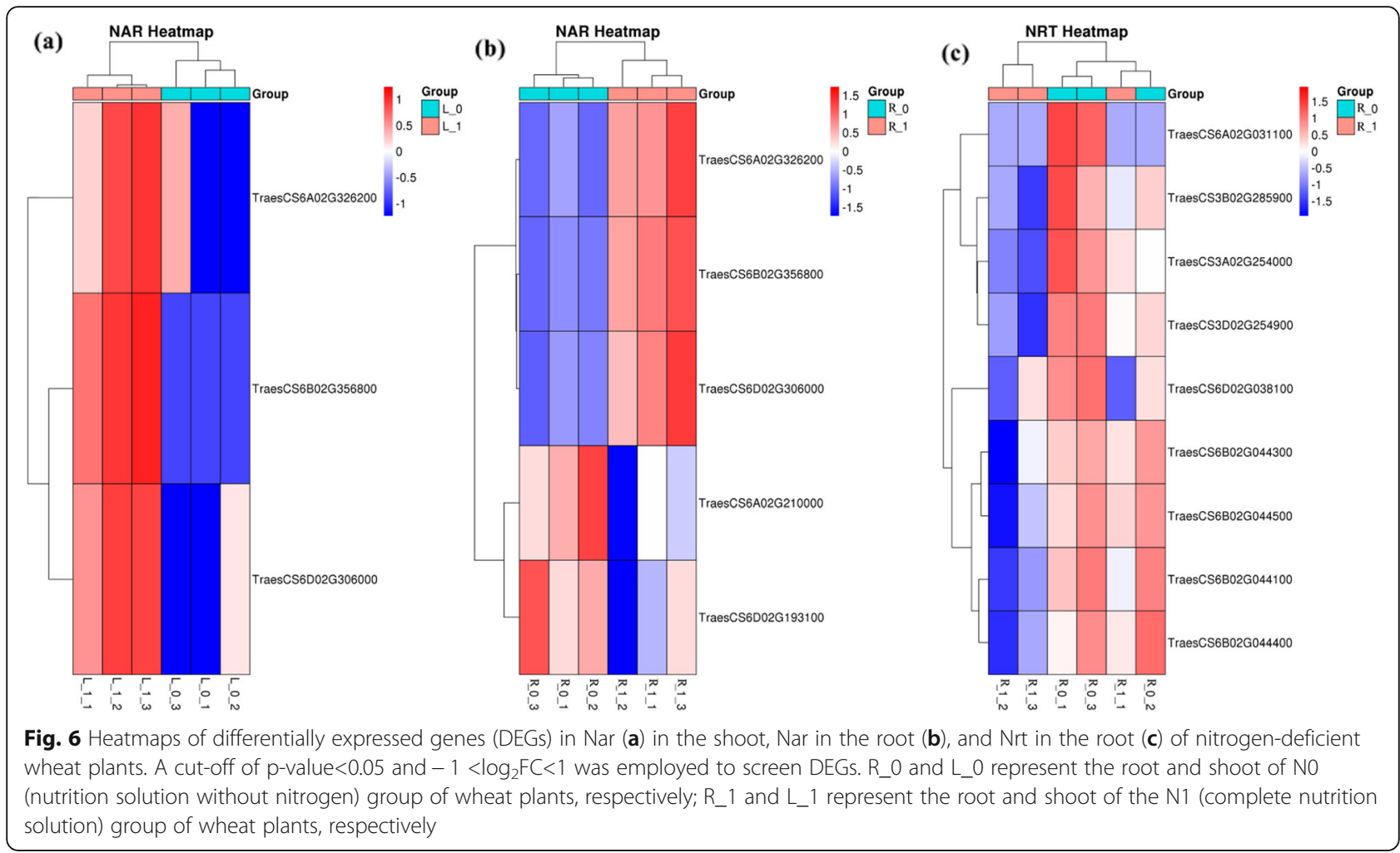

photosynthesis and nitrogen assimilation (Fig. 9). It hampered the biomass accumulation, culminating in reduced shoot height, leaf area, and root volume. Moreover, in the shoot of wheat plants grown under NDC, the downregulated monobactam biosynthesis pathway with the highest enrichment score decreased antibacterial activity. Genetic engineering can be employed to increase the expression of down-regulated genes in four gene families (Pet, Psb, Nar, Nir) identified in our study. It can also increase the rate of photosynthesis and nitrogen metabolism to improve the matter accumulation and growth condition of crops grown under NDC.

\section{Conclusion}

The wheat plants grown under the nitrogen-deficient conditions (NDC) showed reduced crop height, leaf area, root volume, photosynthetic rate, and crop weight and increased root length, root surface area, and root/shoot ratio as compared to control. 3949 (2414 down-regulated, 1535 up-regulated) differentially expressed genes (DEGs) were identified in the shoot, and 3911 (2675 down-regulated, 1536 up-regulated) DEGs were identified in the root of the wheat plants grown under NDC.

GO pathway and KEGG pathway enrichment analysis of these DEGs were also conducted. 24 expansin genes (such as treasCS5B02G528400) and 9 Nrt genes (such as TreasCS6A02G031100) were correlated to increased N absorption. Besides, 3 Pet genes (such as TreasCS7B02G226200) and 8 Psb genes (such as TreasCS3D02G523300) were correlated to the inhibition of the photosynthetic pathway; also, 3 Nar genes (such as TreasCS6A02G326200) and 1 Nir gene (TreasCS6D02G333900) were correlated to the inhibition of nitrogen metabolism pathway in wheat plants grown under NDC.

\section{Methods}

\section{Experimental design}

The nitrogen sensitive wheat cultivar, Shannong 29, was used in this study. The experiments were conducted in the Huang Huai Hai region, China. The seeds of Shannong 29 were procured from ShanDong Shofine Seed Technology Co., Ltd. Two nutrient solution with different nitrogen concentrations $\left(\mathrm{NH}_{4} \mathrm{NO}_{3}\right)$, i.e., complete nutrient solution (N1) with $5 \mathrm{mmol} \mathrm{L}^{-1} \mathrm{NH}_{4} \mathrm{NO}_{3}$ and nutrient solution without nitrogen (N0) with $0 \mathrm{mmol}$ $\mathrm{L}^{-1} \mathrm{NH}_{4} \mathrm{NO}_{3}$ (N0), were used in this study. Thus, wheat plants grown using nutrient solutions, N0 and N1, were referred to as N0 and N1 groups of plants, respectively. Hoagland solution formula was used to prepare a nutrient solution (N0: without nitrogen). The nutrient solution contained $2 \mathrm{mmol} \mathrm{L}^{-1} \mathrm{CaCl}_{2}, 1.8 \mathrm{mmol} \mathrm{L}^{-1} \mathrm{KCl}$, $0.2 \mathrm{mmol} \mathrm{L}^{-1} \mathrm{KH}_{2} \mathrm{PO}_{4}, 0.5 \mathrm{mmol} \mathrm{L}^{-1} \mathrm{MgSO}_{4}, 0.1 \mathrm{mmol}$ $\mathrm{L}^{-1}$ FeEDTA, $0.5 \mu \mathrm{mol} \mathrm{L}^{-1} \mathrm{KI}, \quad 1 \mu \mathrm{mol} \mathrm{L}^{-1} \quad \mathrm{H}_{3} \mathrm{BO}_{3}$, $1 \mu \mathrm{mol} \mathrm{L}^{-1} \quad \mathrm{MnSO}_{4}, \quad 1 \mu \mathrm{mol} \mathrm{L}^{-1} \quad \mathrm{ZnSO}_{4}, \quad 1 \mu \mathrm{mol} \mathrm{L}^{-1}$ $\mathrm{Na}_{2} \mathrm{MoO}_{4}, \quad 0.1 \mu \mathrm{mol} \mathrm{L}^{-1} \mathrm{CuSO}_{4}, \quad 0.1 \mu \mathrm{mol} \mathrm{L}^{-1} \mathrm{CoCl}_{2}$. The $\mathrm{pH}$ was maintained at $6.8 \pm 0.3$. The wheat seeds 


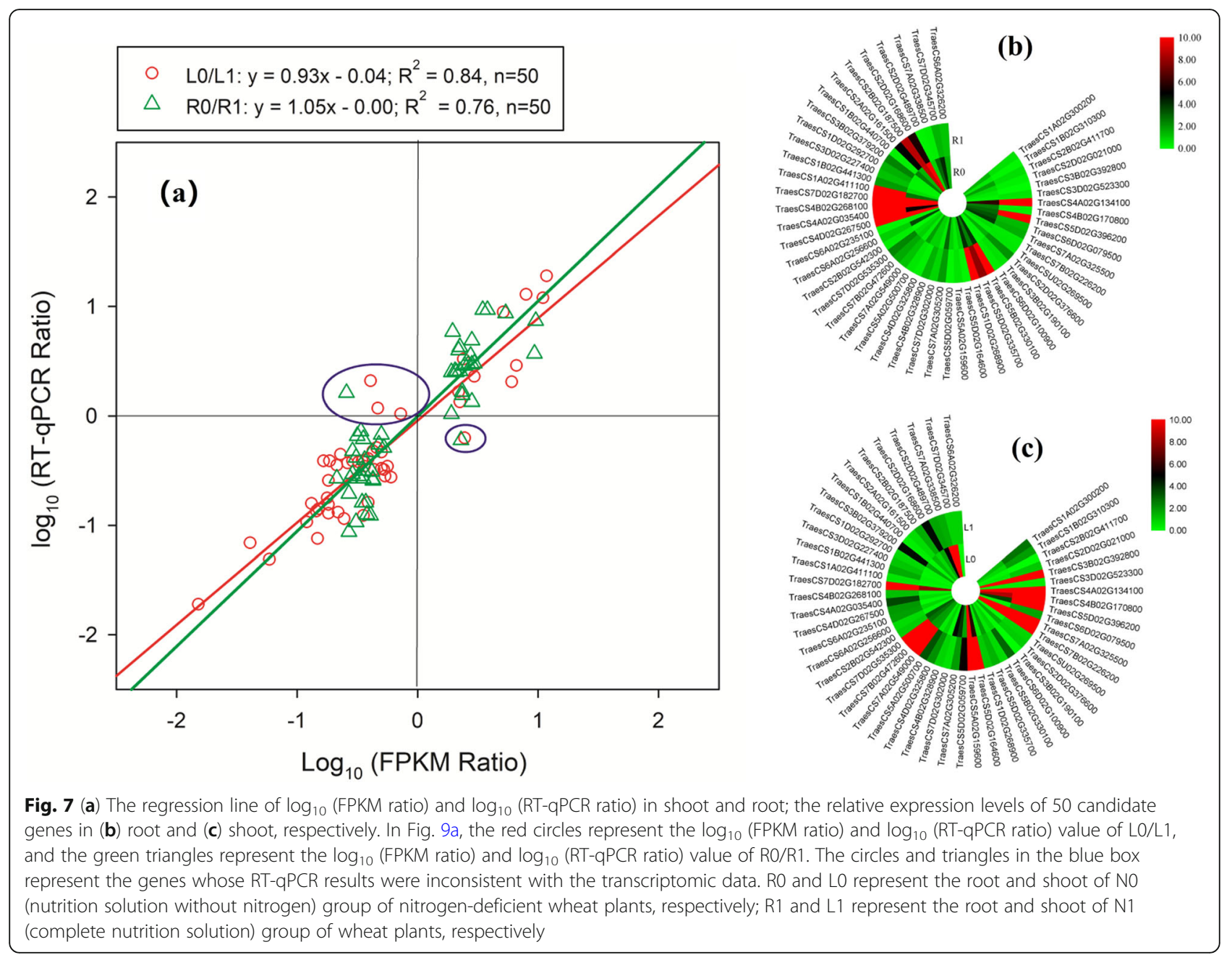

were first sterilized with $75 \%$ alcohol for $30 \mathrm{~s}$ and later washed with sterilized distilled water three times. After sterilizing the winter wheat seeds, the seedlings were cultured to 1 leaf and 1 heart stage. Each experimental treatment contained 100 seedlings, and each experiment was repeated three times. The seedlings were transplanted to different nutrient solutions and fixed by sponges. The seedlings were cultured in an artificial incubator with 8 and $16 \mathrm{~h}$ of dark and light cycle, respectively, and $70 \%$ relative humidity.

\section{Experimental measurements Morphological index}

3 days after transplanting seedlings into nutrient solution, 10 plants in each treatment (repeated in three repetitions) were sampled for measuring leaf area, plant height, root length, root surface area and root volume. In addition, another 10 plants were sampled for determining fresh weight of root and shoot. The method for measuring root length, surface area and volume was followings: artificially rinse the roots, remove impurities and miscellaneous roots, absorb the surface water of the roots, spread the roots in the glass dish of the root scanner $(0.24 \times 0.32 \mathrm{~m})$, and save the photos as $600 \mathrm{API}$ pixels by the root scanner (HP Scanjet 8200; HewlettPackard, Palo Alto, CA, USA). The root analysis software (Delta-T Area Meter Type AMB2; Delta-T Devices Ltd., Cambridge, UK) was used for data analysis.

\section{Physiological index}

3 days after transplanting seedlings into the nutrient solution, the net photosynthetic rate $(\mathrm{Pn})$, stomatal conductance (Gs), and intercellular carbon dioxide concentration $(\mathrm{Ci})$ of top leaves were measured using LI-6400 portable photosynthesizer (LI-COR, USA) with a red-blue light source and a light quantum density of $1400 \mu \mathrm{mol} \mathrm{m} \mathrm{m}^{-1}$.

\section{Transcriptome sequencing}

1 day after transplanting seedlings to the nutrient solution, 20 plants per experimental repetition were quickly sampled, followed by the separation of roots and shoots, 


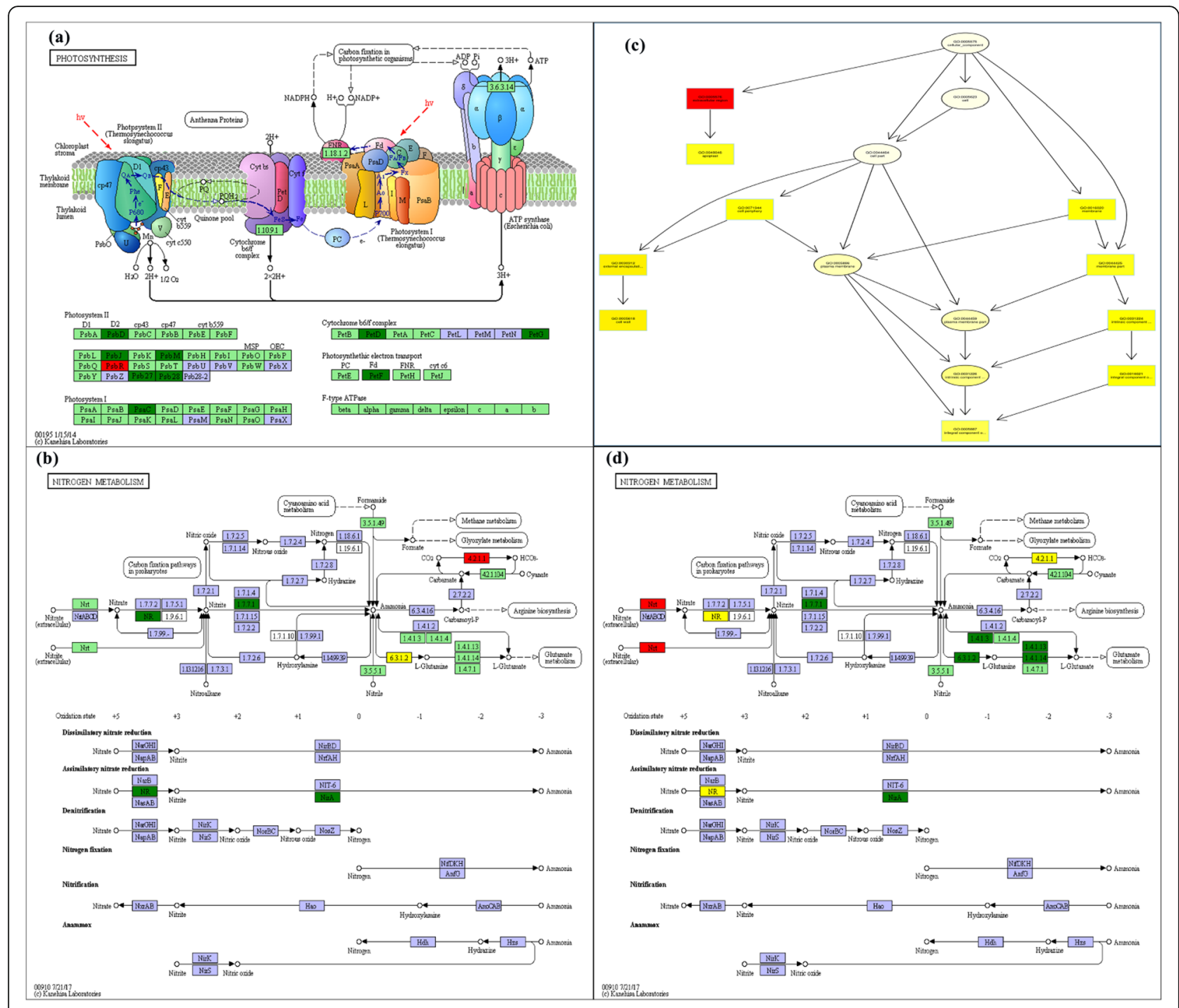

Fig. 8 The KEGG pathway analysis led to the enrichment of DEGs from the wheat shoot in (a) photosynthesis pathway and (b) nitrogen metabolism pathway. The enrichment of DEGs in (b) the extracellular pathway as per the GO analysis, (c) the nitrogen metabolism pathway as per the KEGG analysis (d) identified in the root of the wheat plant. The red frame represents the up-regulated genes; the green frame represents the down-regulated genes

and later these samples were put into liquid nitrogen for quick freezing. Total RNA was extracted using the mirVana miRNA isolation kit (Ambion), as per the manufacturer's instruction. RNA integrity was evaluated using the Agilent 2100 Bioanalyzer (Agilent Technologies, Santa Clara, CA, USA). For subsequent analysis, only the samples with RNA Integrity Number (RIN) $\geq 7$ were used. The libraries were constructed using TruSeq Stranded mRNA LTSample Prep Kit (Illumina, San Diego, CA, USA), according to the manufacturer's instructions. These libraries were sequenced on the Illumina sequencing platform (HiSeqTM 2500 or Illumina HiSeq X Ten), and $125 \mathrm{bp} / 150 \mathrm{bp}$ paired-end reads were generated.

\section{RT-qPCR based validation}

The wheat shoots and roots were sampled at the same time for the transcriptome sequencing. These samples were immediately frozen on liquid nitrogen and stored at $-80^{\circ} \mathrm{C}$. The $50-100 \mathrm{mg}$ plant tissues from each sample were ground into powder in liquid nitrogen, and $500 \mu \mathrm{L}$ buffer RLS was added to each of the powdered samples. The sample was mixed by centrifuge immediately. The RNA was extracted using an RNA kit (Kangwei, China). The PCR reaction mixture contained RNA $7 \mu \mathrm{L}$, Oligo $(\mathrm{dT}) 1 \mu \mathrm{L}$, $2 * \mathrm{R}$ Mix $10 \mu \mathrm{L}$, E-mix $1 \mu \mathrm{L}$, gDNA remover $1 \mu \mathrm{L}$, Rnase-free water $0 \mu \mathrm{L}$. Primers were designed using NCBI's premier blast. The real-time quantitative RT-PCR analysis was carried out by using a multi-channel fluorescent quantitative PCR 


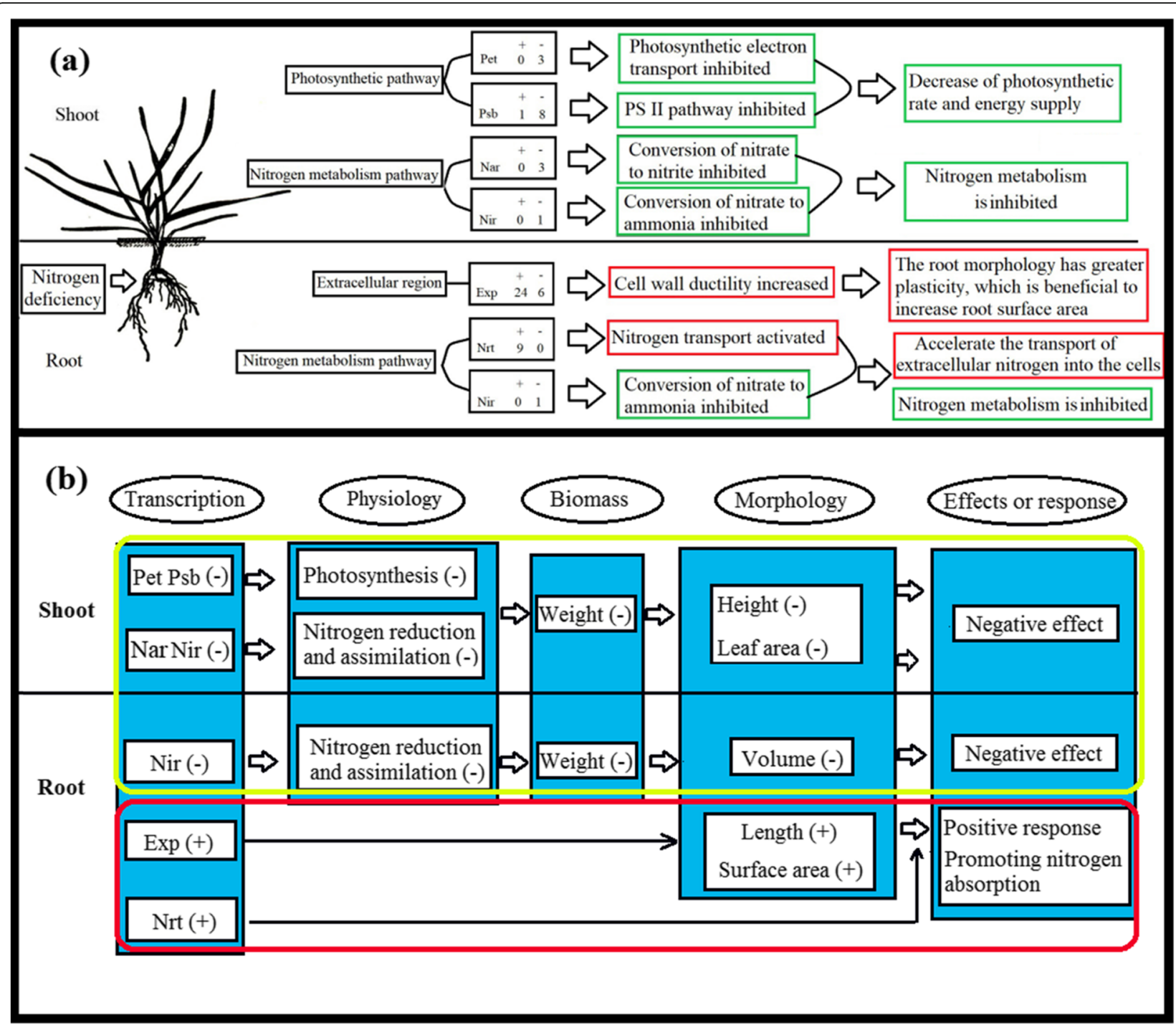

Fig. 9 (a) The chart of pathways as part of plants response to nitrogen-deficient condition; (b) the chart depicts the correlation between gene transcription, physiology, biomass, morphology, and their effects or response under nitrogen deficiency. " + " indicates up-regulated or increased; "-" indicates down-regulated or decreased

instrument (CFX 384 Touch, America). TBtools was employed to construct the heatmaps [45].

\section{Data analysis}

The differentially expressed genes (DEGs) were functionally categorized using Gene Ontology [46]. The genome and mRNA database used was $\mathrm{ftp}: / / \mathrm{ftp}$.ensemblgenomes.org/ pub/plants/release-45/fasta/triticum_aestivum/dna/Triticum_aestivum.IWGSC.dna.toplevel.fa.gz. In GO functional enrichment analysis (http://geneontology.org/ ), all protein-coding genes/transcripts were used as background lists, and differential protein-coding genes/transcripts were used as candidate lists screened from background lists. A hypergeometric distribution test was employed to calculate the $p$ value. It represents the significance of the enriched GO functional category of differential proteincoding genes/transcripts. The p-value was tested using Benjamin \& Hochberg's multiple tests.

We used KEGG [47, 48] database (http://www.genome. $\mathrm{jp} / \mathrm{kegg} /$ ) to analyze the DEGs (combined with KEGG annotation results). Besides, the hypergeometric distribution test was used to calculate the significance of differential gene enrichment in each pathway. The calculated results will return a significant $\mathrm{p}$-value of enrichment, and a small $\mathrm{p}$-value indicated that the differential gene had been 
enriched in the pathway. KEGG pathway enrichment analysis was performed to unveil the enrichment of DEGs.

The experimental data were represented as the mean value from three replicates. Statistical calculations were performed using SPSS software version 19.0 (SPSS Inc., Chicago, USA). Experimental treatments were compared using one-way ANOVA and Duncan's multiple range test (DMRT). $P<0.05$ was considered statistically significant.

\section{Supplementary information}

Supplementary information accompanies this paper at https://doi.org/10. 1186/s12870-020-02662-3.

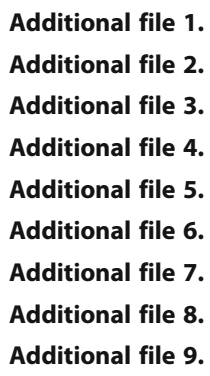

\section{Abbreviations}

Psb: Photosystem II reaction center protein; Pet: Cytochrome b6/f complex subunit 4; Nar: Nitrate reductase; Nrt: High affinity nitrate transporter; Exp: Expansin; Pn: Net photosynthetic rate; Gs: Stomatal conductance; Ci: Intercellular carbon dioxide concentration; DEG: Differentially Expressed Genes; KEGG: Kyoto Encyclopedia of Genes and Genomes; GO: Gene Ontology; NDC: Nitrogen-Deficient Conditions; PCA: Principal Component Analysis; FPKM: Fragments Per Kilobase of transcript per Million fragments mapped

\section{Acknowledgments}

We thank Xingju Zhang in ShanDong Shofine Seed Technology Co., Ltd. for providing the seeds of Shannong 29.

\section{Authors' contributions}

$L X, M Z Q$ and YCM initiated and designed the experiments. $L X, Y C M, X L, J W T$, and XSZ performed the experiments and collected the data. LX analyzed the data and wrote the manuscript. $L X, M Z Q$, and YCM revised the manuscript. All authors read and approved the final manuscript.

\section{Funding}

This work was supported by the National Natural Science Foundation of China (Grant No. 31901457), China Postdoctoral Science Foundation (Grant No. 2019 M652454), Earmarked fund for National Modern Agro-industry Technology Research System of China (Grant No. CARS-27), and Qingchuang Science and Technology Support Project of Shandong Colleges and Universities (Grant No. 2019KJF020).

Purchase of experimental materials, cost of transcriptome data determination, design of the study, interpretation of data, and writing the manuscript were funded by 31901457 and 2019 M652454,

The construction of the experimental platform and purchase of experimental instruments were funded by CARS-27 and 2019KJF020.

\section{Availability of data and materials}

The transcriptome data associated with this article were uploaded for supplementary data. Other datasets are available from the corresponding author on a reasonable request.

Ethics approval and consent to participate Not applicable.
Consent for publication

Not applicable.

\section{Competing interests}

The authors declare that they have no competing interests.

\section{Author details}

${ }^{1}$ State Key Laboratory of Crop Biology, College of Agronomy, Shandong Agricultural University, Taian 271018, Shandong, China. ${ }^{2}$ ShanDong Shofine Seed Technology Co., Ltd., Jiangxiang 272400, Shandong, China. ${ }^{3}$ State Key Laboratory of Crop Biology, College of Horticultural Science and Engineering, Shandong Agricultural University, Taian 271018, Shandong, China.

Received: 17 July 2020 Accepted: 23 September 2020

Published online: 01 October 2020

\section{References}

1. Zhang F, Chen X, Vitousek PM. Chinese agriculture: an experiment for the world. Nature. 2013;497(7447):33-5.

2. Wang $X$, Zhang $H$, Gao $Y$, Zhang W. Characterization of $\mathrm{cu} / Z n-S O D$ enzyme activities and gene expression in soybean under low nitrogen stress. J Sci Food Agric. 2016;96(8):2692-7.

3. Timsina J, Singh U, Badaruddin M, Meisner C, Amin MR. Cultivar, nitrogen, and water effects on productivity, and nitrogen-use efficiency and balance for rice-wheat sequences of Bangladesh. Field Crop Res. 2001;72(2):143-61.

4. Dobermann A, Cassman KG. Plant nutrient management for enhanced productivity in intensive grain production systems of the United States and Asia. Plant Soil. 2002;247(1):153-75

5. Valladares F, Niinemets U. Shade tolerance, a key plant feature of complex nature and consequences. Annu Rev Ecol Evol Syst. 2008;39(1):237-57.

6. Zhu J, Vos J, Der Werf WV, Der Putten PELV, Evers JB. Early competition shapes maize whole-plant development in mixed stands. J Exp Bot. 2014;65(2):641-53.

7. Liu RT, Song C, Su B, Yang F, Yong T, Wu Y, Zhang C, Yang W. Changes in light environment, morphology, growth and yield of soybean in maizesoybean intercropping systems. Field Crop Res. 2017;200:38-46.

8. Han Y, Zhou S, Chen Y, Kong X, Xu Y, Wang W. The involvement of expansins in responses to phosphorus availability in wheat, and its potentials in improving phosphorus efficiency of plants. Plant Physiol Biochem. 2014;78(78):53-62.

9. Sandana P, Pinochet D. Ecophysiological determinants of biomass and grain yield of wheat under P deficiency. Field Crop Res. 2011;120(2):311-9.

10. Liu M, Wu X, Li C, Li M, Xiong T, Tang Y. Dry matter and nitrogen accumulation, partitioning, and translocation in synthetic-derived wheat cultivars under nitrogen deficiency at the post-jointing stage. Field Crop Res. 2020a;248:107720

11. Chen R, Tian M, Wu X, Huang Y. Differential global gene expression changes in response to low nitrogen stress in two maize inbred lines with contrasting low nitrogen tolerance. Gene Genom. 2011:33(5):491-7.

12. Sieger SM, Kristensen BK, Robson CA, Sasan A, Eng EWY, Amal AM, MI M, Vanlerberghe GC. The role of alternative oxidase in modulating carbon use efficiency and growth during macronutrient stress in tobacco cells. J Exp Bot. 2005;416:416

13. Noguchi K, Terashima I. Responses of spinach leaf mitochondria to low N availability. Plant Cell Environ. 2006;29(4):710-9.

14. Noguchi K. Effects of AOX1a deficiency on plant growth, gene expression of respiratory components and metabolic profile under low-Nitrogenn stress in Arabidopsis thaliana. Plant Cell Physiol. 2010:51(5):810-22.

15. Brouillette LC, Donovan LA. Nitrogen stress response of a hybrid species: a gene expression study. Ann Bot. 2011:107(1):101-8.

16. Reddy SK, Liu S, Rudd JC, Xue Q, Payton P, Finlayson SA, Mahan JR, Akhunova A, Holalu SV, Lu N. Physiology and transcriptomics of waterdeficit stress responses in wheat cultivars TAM 111 and TAM 112. J Plant Physiol. 2014;171(14):1289-98.

17. Xanthopoulou A, Ganopoulos I, Psomopoulos F, Manioudaki M, Moysiadis T, Kapazoglou A, Osathanunkul M, Michailidou S, Kalivas A, Tsaftaris A. De novo comparative transcriptome analysis of genes involved in fruit morphology of pumpkin cultivars with extreme size difference and development of EST-SSR markers. Gene. 2017:622:50-66.

18. Liu H, Zhu Q, Pei X, Xing G, Ou X, Li H. Comparative analysis of the photosynthetic physiology and transcriptome of a high-yielding wheat variety and its parents. Crop J. 2020. (In press). 
19. Kawaguchi H, Fukuda I, Shiina T, Toyoshima Y. Dynamical behavior of psb gene transcripts in greening wheat seedlings. I: time course of accumulation of the psbA through psbN gene transcripts during lightinduced greening. Plant Mol Biol. 1992;20(4):695-704.

20. Yi X, Hargett SR, Frankel LK, Bricker TM. The effects of simultaneous RNAi suppression of PsbO and PsbP protein expression in photosystem II of Arabidopsis. Photosynth Res. 2008;98:439-48.

21. Fristedt R, Trotta A, Suorsa M, Nilsson AK, Croce R, Aro E, Lundin B. PSB33 sustains photosystem II D1 protein under fluctuating light conditions. J Exp Bot. 2017;68(15):4281-93.

22. Kato Y, Yokono M, Akimoto S, Takabayashi A, Tanaka A, Tanaka R. Deficiency of the Stroma-lamellar protein LIL8/PSB33 affects energy transfer around PSI in Arabidopsis. Plant Cell Physiol. 2017;58(11):2026-39.

23. Kroliczewski J, Bartoszewski R, Kroliczewska B. Chloroplast PetD protein: evidence for SRP/Alb3-dependent insertion into the thylakoid membrane. BMC Plant Biol. 2017;17(1):213.

24. Monde R, Greene JC, Stern DB. Disruption of the petB-petD intergenic region in tobacco chloroplasts affects petD RNA accumulation and translation. Mol Gen Genomics. 2000;263(4):610-8.

25. Vargassuarez M, Castrosanchez A, Toledoortiz G, La Vara LEGD, Garcia E, Lozatavera $\mathrm{H}$. Protein phosphorylation regulates in vitro spinach chloroplast petD mRNA 3'-untranslated region stability, processing, and degradation. Biochimie. 2013;95(2):400-9.

26. Li Q, Wang X, Ma L, Wei M, Shi Q, Yang F. Molecular characterization of a cucumber nitrate reductase (CsNR) gene under $\mathrm{NO}_{3}$ stress. Mol Biol Rep. 2012;39(4):4283-90.

27. Huarancca Reyes T, Scartazza A, Pompeiano A, Ciurli A, Lu Y, Guglielminetti L, Yamaguchi J. Nitrate Reductase modulation in response to changes in $\mathrm{C} / \mathrm{N}$ balance and nitrogen source in Arabidopsis. Plant Cell Physiol. 2018;59(6):1248-54.

28. Konishi M, Yanagisawa S. Identification of a nitrate-responsive cis-element in the Arabidopsis NIR1 promoter defines the presence of multiple cisregulatory elements for nitrogen response. Plant J. 2010;63(2):269-82.

29. Huang $L$, Zhang $H$, Zhang $H$, Deng $X W$, Wei N. HY5 regulates nitrite reductase 1 (NIR1) and ammonium transporter1;2 (AMT1;2) in Arabidopsis seedlings. Plant Sci. 2015;238:330-9.

30. Zhao L, Zhang W, Yang Y, Li Z, Li N, Qi S, Crawford NM, Wang Y. The Arabidopsis NLP7 gene regulates nitrate signaling via NRT1.1 -dependent pathway in the presence of ammonium. Sci Rep. 2018;8(1):1487.

31. Ho C, Frommer WB. Fluorescent sensors for activity and regulation of the nitrate transceptor CHL1/NRT1.1 and oligopeptide transporters. eLife. 2014;3:01917.

32. Bouguyon E, Perrinewalker F, Pervent M, Rochette J, Cuesta C, Benkova E, Martiniere A, Bach L, Krouk G, Gojon A. Nitrate controls root development through posttranscriptional regulation of the NRT1.1/NPF6.3 transporter/ sensor. Plant Physiol. 2016;172(2):1237-48.

33. Teng $Y$, Liang $Y$, Wang M, Mai H, Ke L. Nitrate transporter 1.1 is involved in regulating flowering time via transcriptional regulation of FLOWERING LOCUS C in Arabidopsis thaliana. Plant Sci. 2019;284:30-6.

34. Zhang X, Cui Y, Yu M, Su B, Gong W, Baluska F, Komis G, Samaj J, Shan X, Lin J. Phosphorylation-mediated dynamics of nitrate Transceptor NRT1.1 regulate Auxin flux and nitrate signaling in lateral root growth. Plant Physiol. 2019;181(2):480-98.

35. Fang $X Z$, Tian $W H$, Liu XX, Lin XY, Jin C, Zheng SJ. Alleviation of proton toxicity by nitrate uptake specifically depends on nitrate transporter 1.1 in Arabidopsis. New Phytol. 2016;211(1):149-58.

36. Jian S, Liao Q, Song H, Liu Q, Lepo JE, Guan C, Zhang J, Ismail AM, Zhang Z. NRT1.1-related NH4 + toxicity is associated with a disturbed balance between NH4 + uptake and assimilation. Plant Physiol. 2018;178(4):1473-88.

37. Medici A, Szponarski W, Dangeville P, Safi A, Dissanayake IM, Saenchai C, Emanuel A, Rubio V, Lacombe B, Ruffel S. Identification of molecular integrators shows that nitrogen actively controls the phosphate starvation response in plants. Plant Cell. 2019:31(5):1171-84.

38. Gao Q, Zhao M, Li F, Guo Q, Xing S, Wang W. Expansins and coleoptile elongation in wheat. Protoplasma. 2008;233:73-81.

39. Ilias IA, Negishi K, Yasue K, Jomura N, Morohashi K, Baharum SN, Goh HH. Transcriptome-wide effects of expansin gene manipulation in etiolated Arabidopsis seedling. J Plant Res. 2019;132(2):159-72.

40. Han Z, Liu Y, Deng X, Liu D, Liu Y, Hu Y, Yan Y. Genome-wide identification and expression analysis of expansin gene family in common wheat (Triticum aestivum L.). BMC Genomics. 2019;20(1):1-19.
41. Li F, Xing S, Guo Q, Zhao M, Zhang J, Gao Q, Wang G, Wang W. Drought tolerance through over-expression of the expansin gene TaEXPB23 in transgenic tobacco. J Plant Physiol. 2011;168(9):960-6.

42. Peng $L, X u$ Y, Wang $X$, Feng $X$, Zhao Q, Feng S, Zhao Z, Hu B, Li F. Overexpression of paralogues of the wheat expansin gene TaEXPA8 improves low-temperature tolerance in Arabidopsis. Plant Biol. 2019;21(6):1119-31.

43. Yu X, Wang T, Zhu M, Zhang L, Zhang F, Jing E, Ren Y, Wang Z, Xin Z, Lin T. Transcriptome and physiological analyses for revealing genes involved in wheat response to endoplasmic reticulum stress. BMC Plant Biol. 2019;19(1):1-22.

44. Kumar J, Gunapati S, Kianian SF, Singh SP. Comparative analysis of transcriptome in two wheat genotypes with contrasting levels of drought tolerance. Protoplasma. 2018;255(5):1487-504.

45. Chen C, Chen H, Zhang Y, Thomas HR, Xia R. TBtools: An Integrative Toolkit Developed for Interactive Analyses of Big Biological Data. Mol Plant. 2020;13(8):1194-202.

46. Thomas PD. The gene ontology and the meaning of biological function. Method Mol Biol. 2017:1446:15-24.

47. Kanehisa M, Sato Y, Kawashima M, Furumichi M, Tanabe M. KEGG as a reference resource for gene and protein annotation. Nucleic Acids Res. 2016:44:457-62.

48. Kanehisa M, Furumichi M, Tanabe M, Sato Y, Morishima K. KEGG: new perspectives on genomes, pathways, diseases and drugs. Nucleic Acids Res. 2017;45:D353-61.

\section{Publisher's Note}

Springer Nature remains neutral with regard to jurisdictional claims in published maps and institutional affiliations.
Ready to submit your research? Choose BMC and benefit from:

- fast, convenient online submission

- thorough peer review by experienced researchers in your field

- rapid publication on acceptance

- support for research data, including large and complex data types

- gold Open Access which fosters wider collaboration and increased citations

- maximum visibility for your research: over $100 \mathrm{M}$ website views per year

At BMC, research is always in progress.

Learn more biomedcentral.com/submissions 